The PDF as originally published does not show the correct project number. This research was supported by a grant from the Hong Kong Polytechnic University (project number J-BB9M) and the University of Hong Kong Strategic Research Theme of Information Technology. 


\title{
Wavelength-Routed Networks With Lightpath Data Interchanges
}

\author{
C. Y. Li, Lin Cai, P. K. A. Wai, Victor O. K. Li, and Anshi Xu
}

\begin{abstract}
We observe that tunable wavelength converters (TWCs) that are traditionally installed in wavelength-routed (WR) networks for wavelength contention resolution can be further utilized to provide fast data switching between lightpaths. This allows us to route a data unit through a sequence of lightpaths from source to destination if a direct single lightpath connection is not available or if we want to minimize the overhead of setting up new lightpaths. Since TWCs have a tuning time of picoseconds, it may be possible to use the installed TWCs as lightpath data interchanges (LPIs) to improve the performance of WR networks without significant optical hardware upgrade. Compared with the multihop electronic grooming approach of lightpath networks, the LPI approach has a simpler WR node architecture, does not need expensive high-speed electrical multiplexers/routers, and does not sacrifice the bitrate/format transparency of data between the source and destination. Our simulation results show that WR networks with LPIs can have much lower blocking probability than WR networks without LPIs if the traffic duration is short. We show that LPIs can also be used to provide new data transportation services such as optical time division multiplexing access (OTDMA) time-slotted service in WR networks.
\end{abstract}

Index Terms-Wavelength-routed networks; Tunable wavelength converters; Wavelength division multiplexing; Optical time division multiplexing.

\section{INTRODUCTION}

$\mathbf{O}$ ptical networks have become an indispensible infrastructure to satisfy the growing traffic demand. Hundreds of high-speed wavelength channels can be used for data transmission in a single optical fiber using wavelength division multiplexing (WDM)

Manuscript received December 7, 2009; revised May 8, 2010; accepted May 19, 2010; published June 9, 2010 (Doc. ID 121107).

C. Y. Li (e-mail: cyli@eie.polyu.edu.hk) and P. K. A. Wai are with the Department of Electronic and Information Engineering, The Hong Kong Polytechnic University, Hung Hom, Kowloon, Hong Kong.

L. Cai and A. Xu are with Peking University, Beijing, China.

V. O. K. Li is with The University of Hong Kong, Hong Kong.

Digital Object Identifier 10.1364/JOCN.2.000415 technology. Although WDM technology can effectively utilize the $25,000 \mathrm{GHz}$ bandwidth of optical fibers, the electronics inside the network nodes are becoming the bottleneck of data transmission [1,2]. Consequently, lightpaths in wavelength-routed (WR) networks have been proposed to provide all-optical transmission between the source and destination nodes [3,4]. A lightpath reserves a wavelength channel at each optical fiber link on the path before communication between the source and destination nodes can begin. No optical-to-electrical-to-optical (OEO) conversion and no processing of the data are required at any intermediate node during the transmissions. This simplifies WR nodes and eliminates the need for expensive highspeed electrical routers. Since the bit-rate/format transparency of data is guaranteed, many services such as IP over WDM [5], video on demand [6], and multicasting [7] have been proposed on WR network platforms.

WR networks using lightpaths can provide large transmission bandwidth in a comparatively economical way, but a lightpath by nature is not designed for supporting data traffic with bursty characteristics $[8,9]$. Owing to the end-to-end acknowledgment delay and the millisecond reconfiguration time of optical switches in intermediate nodes, the setup/teardown requirement makes lightpaths suitable only for data traffic of long duration. Since bursty traffic can have a duration much shorter than the lightpath setup time, the utilization of transmission bandwidth will be low if only the lightpath approach is used for data transmission in WR networks. Consequently, a sequence of lightpaths instead of a single lightpath is proposed to connect a node pair $[10,11]$. Such approaches permit us to electrically add/drop traffic at the nodes connecting the lightpaths. Traffic to the same destination can be electrically multiplexed (groomed) into the lightpath at such intermediate nodes. This increases the lightpath transmission bandwidth utilization and reduces the required number of lightpaths in the networks. The disadvantages of such approaches are more complicated WR nodes, additional expensive high-speed electrical multiplexers/routers, and sacrificing the bit-rate/format transparency of data between the sources and destinations. 
Sophisticated optical data transportation schemes such as optical packet switching (OPS) $[1,2,12,13]$ and optical burst switching (OBS) [14-17] have been proposed to handle bursty traffic, but most of them are still in the research stage. Some of the proposed schemes, including OPS, require technologies such as all-optical packet buffering that will not be available soon [1,2]. Other schemes such as OBS can implement its basic model using present technology, but the implementation of a high-performance model also requires technologies similar to that of OPS. On the other hand, most of the required technologies for WR networks are already available [18-20]. WR networks are likely to be the dominant optical network infrastructure in the near future. To protect the huge investment in infrastructure, we need new optical data transportation schemes that can reuse the WR networks to provide fast data switching services.

At first glance, additional fast optical switching devices are required in WR networks to support fast data switching applications because slow optical switches with millisecond reconfiguration time are usually used in WR nodes for the setup/teardown of lightpaths [21-23]. However, optical switches are not the only devices used for data exchange in WR networks. To resolve wavelength contentions between lightpaths when they are set up, tunable wavelength converters (TWCs) have been used in WR networks to convert the wavelength of a data transmission link by link along the lightpath [4]. We observe that once a lightpath is set up, in normal lightpath applications, the TWCs associated with the lightpath will not be adjusted until the lightpath is taken down. This is to prevent a data unit from being misrouted to a different lightpath and hence to the wrong destination. Evidently, we can forward data units from a source to different destinations without changing the optical switch settings or without electrically switching the data units at intermediate nodes if we can properly adjust the TWCs to change the data units from one lightpath to another after the lightpath setup. TWCs in WR networks can not only resolve wavelength contention but can also be further used as the interchange of data units between lightpaths, i.e, lightpath data interchange (LPI).

Applying LPI in WR networks requires us to accurately coordinate the data switching between wavelengths. It will first require a synchronization mechanism for the data switching, which is still challenging because of the technical difficulties posed by physical impairments from the fiber layer such as dispersion. In spite of this, the extensively studied time-slotbased optical networks can provide useful experience for implementing the synchronization between LPIs. As all-optical wavelength conversion technologies are available, TWCs can be bit-rate/format transparent of data and can have picosecond reconfiguration (tuning) time [24-26]. Using TWCs as LPIs can provide fast data switching/forwarding capability that is not available in the normal lightpath WR networks.

In this paper, we study the performance of using TWCs for LPI in WR networks when fast data switching is provided. In Section II, we will first review the node structure of WR networks and then describe the proposed WR networks with LPIs in detail. For WR networks without TWCs or with slow TWCs, external LPI devices will have to be introduced. Simulations show that WR networks with TWCs additionally supporting LPI function can have much better system performance than the normal WR networks if the traffic duration is short. In Section III, we show that the proposed LPI can also be used to provide new data transportation services such as optical time division multiplexing access (OTDMA) time-slotted service on WR networks. By using OTDMA, a wavelength channel can be further divided into time-slotted subchannels to improve the bandwidth utilization. Using LPIs, data in different time slots of the same wavelength channel can be routed to destinations on different lightpaths. Hence, the transmission bandwidth utilization is further improved. From simulation results, we show that WR networks with LPIs can have significant performance improvement over WR networks without LPIs if time-slotted subchannel services are provided. Section IV concludes the paper.

\section{WR NETWORKS With LightPATH DATA INTERCHANGE}

Figure 1, shows a WR node structure with TWCs installed at the inputs of the optical switch. The control circuits are omitted to simplify the illustration. In Fig. 1 , there are $K 1$-to- $W$ wavelength demultiplexers (DEMUXs), $K W$ TWCs, a $K W \times K W$ optical switch (SW), and $K W$-to- 1 optical combiners. If only lightpath applications are considered, wavelength multiplexers can be used to replace the optical combiners in Fig. 1. Wavelength multiplexers have the advantage of signal

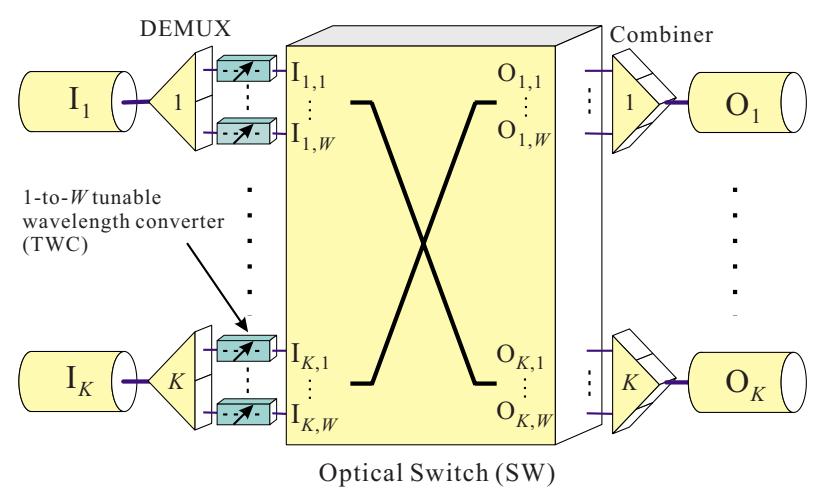

Fig. 1. (Color online) WR node with TWCs installed. 
power loss being insensitive to the number of input ports [27]. However, most available wavelength multiplexers have strict wavelength-input requirements, e.g., an optical signal of wavelength $\lambda_{1}$ will not be transferred to the output if it is not sent through input 1 of the wavelength multiplexer. In contrast, an optical combiner transfers an optical signal from any one of its inputs to its output irrespective of the wavelength of the optical signal. LPI will take advantage of this feature of optical combiners. The signal power loss of most commercially available passive optical combiners grows with the number of inputs. However, low-signal-power-loss and even zero-loss passive optical combiners have been recently proposed [28,29]. In this paper, we assume that active optical combiners integrated with the signal amplification function will be used if $W$ is large [30].

In Fig. 1, the optical signal of input fiber $I_{j}$ is demultiplexed into $W$ wavelengths $\lambda_{1}$ to $\lambda_{W}$ and sent to input $\mathrm{I}_{j, 1}$ to input $\mathrm{I}_{j, W}$ of the optical switch SW. In general, the optical switch SW is internally nonblocking with a reconfiguration time of milliseconds or longer. An optical signal from any input $\mathrm{I}_{j, k}$ can be connected to any free output $\mathrm{O}_{x, y}$ of the switch SW. As the data will be corrupted if two optical signals with the same wavelength are sent to the same output optical fiber, the TWCs in Fig. 1 convert the optical signals into proper wavelengths to avoid wavelength contention before sending the signals into the optical fibers. Otherwise, a wavelength $\lambda_{x}$ optical signal at the $\mathrm{SW}$ input $\mathrm{I}_{j, x}$ can only be sent to one of the $K$ outputs $\mathrm{O}_{1, x}$ to $\mathrm{O}_{K, x}$ even if SW is internally nonblocking. Apart from resolving the wavelength contentions between lightpaths, the TWCs in Fig. 1 also give us flexible wavelength channel assignment in lightpath communications. In WR networks, a lightpath is required to reserve a wavelength channel on each link of the path before communications can begin between two nodes. Figure 2 shows a four-node WR network with two wavelength channels $\left(\lambda_{1}\right.$ and $\left.\lambda_{2}\right)$ per link, and four lightpaths $\mathrm{P}_{1}$ to $\mathrm{P}_{4}$ passing through the nodes. The lightpaths in Fig. 2 use the same wavelength channel in all links of their

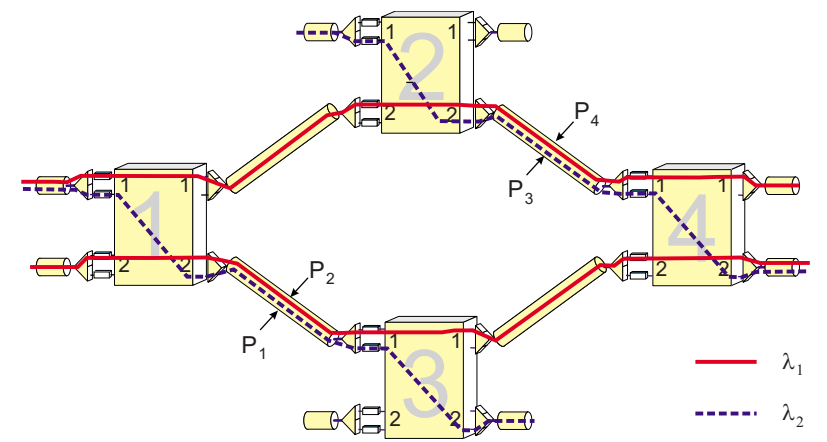

Fig. 2. (Color online) Four-node wavelength-routed network with two wavelength channels per link. corresponding routes but this is not necessary if the WR nodes of Fig. 1 are used. With the TWCs installed in the WR nodes, we have the flexibility to use different wavelength channels in different links for a lightpath. Hence, WR networks with TWCs installed in the nodes often have much better call blocking performance. Optimizing the utilization of TWCs such as minimizing the number of required TWCs in a node and/or in the whole network has become an important research topic in WR networks [4].

The application of TWCs so far has not changed the principle of data communications with lightpaths in WR networks. To avoid OEO conversion and electrical processing at intermediate nodes, two nodes in WR networks still have to establish a lightpath between them for data communications. Data are transported along the established lightpath like a pipe from source to destination even though the lightpath may be composed of segments of different wavelength channels. Owing to the millisecond optical switch reconfiguration time and the large end-to-end signal acknowledgment propagation time, frequent setup and teardown of lightpaths should be avoided to reduce the overhead. However, a lightpath can also be severely underutilized if short duration traffic is carried in the lightpath. For example, we may need to set up $M N$ lightpaths between all source-destination pairs in Fig. 3 if the intertraffic time gaps inside all the lightpaths are shorter than the optical switch reconfiguration time, though the total traffic volume is much smaller than $M N$ erlangs. As traffic becomes bursty and the fiber transmission rate increases, WR networks relying on lightpath communications will not be able to handle the increasing short duration traffic.

\section{A. Proposed Lightpath Data Interchange}

In general, fast optical switching devices are required if we want to have high bandwidth utilization for short duration traffic. However, since the optical switches in WR nodes are installed for lightpath applications, their reconfiguration time is typically milliseconds or longer. Upgrading the slow optical switches with faster ones may not be feasible. Note that the optical switches in the WR nodes can have a thousand input/output ports [21]. At the moment, fast

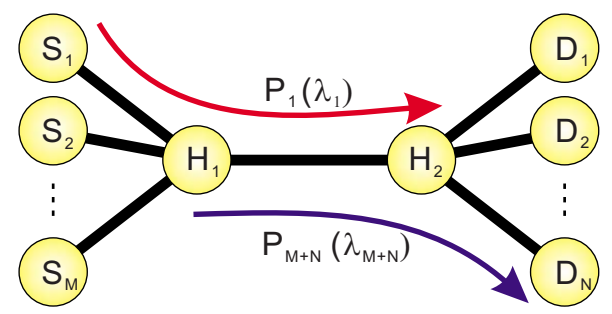

Fig. 3. (Color online) WR network with $M$ source and $N$ destination nodes. 
optical switches are only available in small sizes such as $2 \times 2[22,23]$. Even if the required large and fast optical switches become available, upgrading the switches in WR nodes will still be very costly. We observe that it is not necessary to modify the existing WR nodes to provide fast data switching services if the TWCs originally used to resolve wavelength contentions at lightpath setup can also be used for switching data between lightpaths.

A lightpath is defined by a sequence of fiber segments and the wavelength channels within each segment connecting the source and destination. The states of the TWCs on a lightpath are set to the appropriate values as required by the lightpath during lightpath setup and remain unchanged during data transmission [4]. Data are transported along the lightpath from source to destination passing through a series of TWCs and are converted to the predefined wavelengths at each of the TWCs. Thus if data following a particular lightpath are converted by a TWC on the lightpath to a wavelength different from that prescribed by the lightpath, then the data will drop off the lightpath. The misrouted data may be discarded or in the worst case be routed into another lightpath and interfere with the data transmitting on that lightpath. However, if the data collisions are avoided by coordinating the data transmissions in the lightpaths, data at a TWC on a particular lightpath can be switched to another lightpath by converting the data to the wavelength of the new lightpath instead of the original one. In other words, the TWCs in WR networks can also serve as data switches in the wavelength domain. Data communications will not need a direct lightpath connecting the source and destination as long as a sequence of lightpaths can be found connecting the node pairs. Thus, without additional fast optical switching devices, the TWCs in WR nodes can be used as LPIs to switch data between lightpaths as they travel to their destinations.

Figure 2 shows an example of a WR network with LPIs. There are four lightpaths passing the nodes of Fig. 2. We cannot set up new lightpaths from node 1 and node 2 to any output of node 4 because there is no free wavelength channel available in links (1-3) and (2-4). Using the normal lightpath approach, sending data from input 2 of node 1 to output 2 of node 3 (input 1 of node 2 to output 1 of node 4 ) is not possible unless one of the two lightpaths $\mathrm{P}_{1}$ and $\mathrm{P}_{2}\left(\mathrm{P}_{3}\right.$ and $\left.\mathrm{P}_{4}\right)$ is torn down. Using the LPI approach, however, data can enter lightpath $\mathrm{P}_{2}\left(\mathrm{P}_{3}\right)$ at input 2 of node 1 (input 1 of node 2), move to lightpath $\mathrm{P}_{1}\left(\mathrm{P}_{4}\right)$ at output 2 of node 1 (output 2 of node 2 ) by changing its wavelength to $\lambda_{2}\left(\lambda_{1}\right)$, and finally depart at output 2 of node 3 (output 1 of node 4 ). Hence, no new lightpath setup is required. We have assumed that the spare transmission capacities of lightpath $\mathrm{P}_{1}$ to lightpath $\mathrm{P}_{4}$ are suffi- cient, and the traffic transmissions of different lightpaths are properly coordinated. Owing to its importance, coordination of traffic transmissions of different lightpaths will be discussed in more detail in Subsection II.C.

In the example of Fig. 3 , the path between nodes $\mathrm{S}_{i}$ and $\mathrm{D}_{j}$ has three hops. The two nodes can be connected using the LPI approach with two two-hop lightpaths $\mathrm{P}_{i}$ and $\mathrm{P}_{M+j}$. The link between $\mathrm{H}_{1}$ and $\mathrm{H}_{2}$ is the intersection link of lightpaths $\mathrm{P}_{i}$ and $\mathrm{P}_{M+j}$. The examples of Figs. 2 and 3 illustrate how the LPI approach can be used to route data through a sequence of lightpaths from source to destination such that a direct lightpath connection is not necessary. The requirement of the valid sequence of $H$ lightpaths for LPI data routing is rather simple:

1. The first and the $H$ th lightpaths on the sequence are connected to the source and destination nodes, respectively.

2. Adjacent lightpaths on the sequence must have one or more intersection links.

3. For $H=3$, the intersection links of the $j$ th and $(j+1)$ th lightpaths will be on the path downstream from the intersection links of the $(j$ -1 )th and $j$ th lightpaths, where $j=2,3, \ldots, H-1$. From the above lightpath sequence requirements, all lightpaths will have path lengths of at least two hops. The resultant routing path between source and destination using the LPI approach will have a path length of at least three hops.

One advantage of using LPI is to relax the requirement of lightpath setup/teardown by transmitting a data unit lightpath by lightpath. This advantage will become important if the traffic duration $T_{d}$ is short and comparable with the reconfiguration time of the optical switch $T_{s w}$ since TWCs can have tuning time $T_{w c}$ down to picoseconds $[22,24,26]$. Figure 4 shows the busy periods of the SW, TWC, and output link $\left(\mathrm{O}_{1}\right)$ of the last intermediate WR node on a lightpath during a data transmission cycle. A source node in general has to confirm that all nodes on the path are ready before transmitting data, i.e., the two-way resource reservation of circuit switching. Time period $t_{4}$ to $t_{5}$ is therefore the round-trip time $T_{r t}$ between the

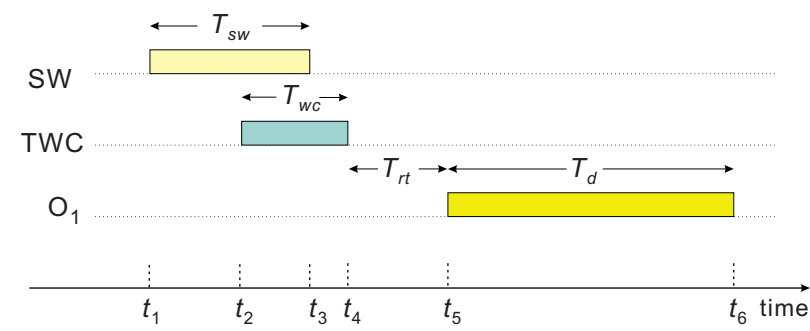

Fig. 4. (Color online) Busy periods of the SW, TWC, and output link $\left(\mathrm{O}_{1}\right)$ of the last intermediate WR node on a lightpath during a data transmission cycle. 
source and the WR node. As $T_{w c}$ is generally much smaller than $T_{s w}$ and TWC can start tuning before the completion of SW reconfiguration, $t_{4}$ can even be smaller than $t_{3}$ if SW and TWC are properly scheduled. Nevertheless, although the actual data transmission on $\mathrm{O}_{1}$ is only from time $t_{5}$ to $t_{6}, \mathrm{O}_{1}$ is unavailable to other lightpaths from time $t_{1}$ to $t_{6}$. Any new lightpath setup requiring $\mathrm{O}_{1}$ will be blocked during this time period. The time period from $t_{1}$ to $t_{5}$ is a fixed overhead for all data transmissions and will become significant if the transmission duration $T_{d}$ is small. Note that $T_{r t}=1 \mathrm{~ms}$ means $100 \mathrm{~km}$ distance between the source and the WR node. If the link distance between nodes is small, $T_{s w}$ will constitute the majority of the overhead. Transporting data on a sequence of established lightpaths will have much smaller overhead because no optical switch reconfiguration is required.

With LPIs, a WR network can choose to set up a smaller number of lightpaths although this is not the main purpose of using LPIs. For example, in Fig. 3, we only need to set up $M$ lightpaths from nodes $\mathrm{S}_{i}$ to $\mathrm{H}_{2}$ using the wavelength channels of $\lambda_{i}, i=1, \ldots, M$, and $N$ lightpaths from nodes $\mathrm{H}_{1}$ to $\mathrm{D}_{j}$ using the wavelength channels of $\lambda_{M+j}, j=1, \ldots, N$, if LPIs are used and the total traffic at each link does not exceed the capacity.

\section{B. Different Implementations of WR Networks With LPIS}

For wavelength contention resolution purposes, it makes no difference whether the TWCs are installed at the inputs or outputs of the WR nodes. For LPI applications, however, installing TWCs at the WR node inputs seems to have an advantage because the original 1-to- $W$ TWCs used for wavelength contention resolution can also serve as the required LPIs unless the tuning time is larger than the LPI requirement. If output TWCs are used, the TWCs will be $W$-to- 1 type or OEO type fixed output WCs. In either case, we will need to replace the output TWCs by $W$-to- $W$ type TWCs or use an external LPI device.

Figure 5 shows an alternative implementation of

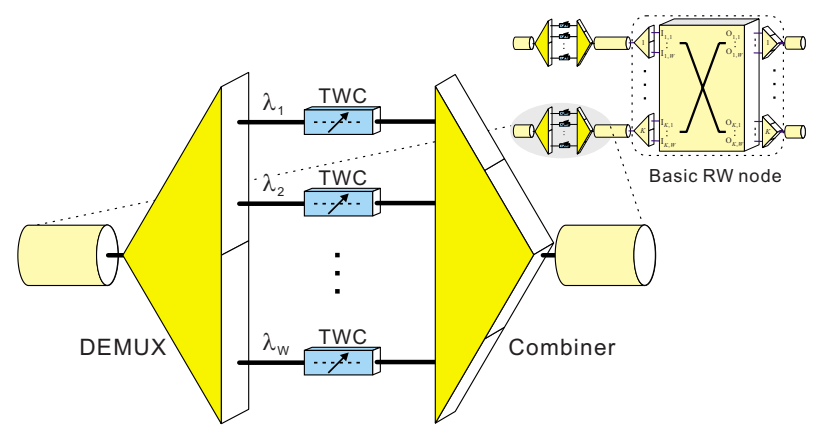

Fig. 5. (Color online) External LPI device.
LPI to WR networks by adding an external LPI device at the input port of a WR node. The wavelengths of the data are reassigned before the data are sent to the WR node. Hence, interchanging data between lightpaths will be independent of the architecture of the WR nodes. It is expected that the first WR networks are constructed from basic WR nodes that have no internal TWC. In some basic WR node implementation approaches such as broadcast-and-select [22], the individual wavelength signal may not even be accessible for wavelength conversion. Upgrading such basic WR nodes to have TWCs for wavelength contention resolution will be expensive and difficult. The external LPI device will be useful in such situations because the wavelength conflict can also be solved externally at the external LPI devices when the lightpaths are set up. No modification is required for the basic WR nodes [31].

Compared with a WR node with preinstalled TWCs, the combination of a basic WR node and external LPI devices put more limitations on lightpath setup. For example, we can reserve an idle node output wavelength channel $\lambda_{x}$ for a lightpath only if the $\lambda_{x}$ wavelength channel between the external LPI device and the node input is not occupied by other lightpaths. However, the use of external LPI devices maximizes the flexibility of the deployment of WR networks. Since the performance of the internal LPI will obviously be better than or equal to that of the external one, in this paper, all performance evaluation of WR networks with LPIs focus on combinations of basic WR nodes with external LPI devices.

Figure 1 does not show the local traffic add-drop ports of the WR node. One approach of adding the local ports to the WR node is to connect the local ports to the WR node through the optical switch SW. Using this method, however, we need to replace SW by a $2 K W \times 2 K W$ optical switch. Another way is to install $K W 1 \times 2$ drop switches at the inputs of the SW and $K W 2 \times 1$ add switches at the outputs of the SW. With this approach, there is no need to use a larger optical switch for SW. Since fast optical switches are available in small sizes such as $1 \times 2$ and $2 \times 1$, we may further use fast add-drop switches to shorten the lightpath setup/teardown time. If external LPI devices are used, the $1 \times 2$ drop switches should be installed at the outputs of the DEMUX in Fig. 5 instead of at the inputs of the optical switch in a basic WR node. Otherwise, the recovered wavelength channel capacity from the locally dropped traffic will not be immediately available for other traffic passing through the external LPI device. The performance improvement with the fast traffic add-drop is more significant in WR networks with LPIs than WR networks without LPIs. 


\section{Coordination of Traffic Transmission of Different Lightpaths}

The performance of LPIs highly depends on the availability of sequences of lightpaths that can be used to connect the source and destination. Ideally, we can dynamically suspend/resume all transmissions in existing lightpaths when a new transmission (call) happens. For example, in Fig. 3, we will need to temporarily suspend the transmission from $\mathrm{H}_{1}$ to $\mathrm{D}_{N}$ when $\mathrm{S}_{1}$ is sending data to $\mathrm{D}_{N}$ if $\mathrm{H}_{1}$ is not a pure routing node. This approach of using LPI can maximize the benefit from LPI, but it requires new sophisticated control signaling and transmission scheduling schemes on top of the lightpaths. On the other hand, if we have no control over the transmission in existing lightpaths but can predict all idle periods of the lightpaths (assuming the transmission bandwidth of lightpaths is not fully utilized), another approach of using LPI is to schedule new transmissions in the idle periods. One example of such a network is realized by the application of OTDMA on WR networks. We will discuss it further in Section III.

Occasionally, we may not be able to know the transmission status of existing lightpaths. To take advantage of LPI, we should arrange temporary lightpaths (with no traffic transmission) in WR networks to reduce the need to set up new lightpaths for new calls. A simple way to do this is to maintain the optical switch configurations for a lightpath after the completion of the traffic transmission until there is a conflict with the lightpath requirement of a new call. To have better system performance, however, we need to predict future traffic fluctuation when maintaining such temporary lightpaths. Numerous traffic prediction techniques have been proposed for routing and resource scheduling [32,33]. The derivation of a proper scheme for temporary lightpath preparation will be straightforward if the appropriate traffic prediction technique has been identified.

\section{Blocking Performance of WR Networks With LPIs}

Figures 6-9 show the comparison between the blocking probabilities of WR networks with and without LPIs and with and without the fast traffic adddrop function. In the simulations, the traffic duration $T_{d}$ is chosen to be a negative exponential random variable with a mean of one time unit. The call arrival process is Poisson. When a call arrives at a source node, it chooses a node at random for its destination. The fixed shortest path is used in the simulations. There are 16 wavelength channels per fiber. The source-to-destination end-to-end propagation delay in Figs. $6-8$ is assumed to be negligible, whereas that of Fig. 9 is not. When it is necessary to reconfigure the optical switches and the reconfiguration time $T_{s w}$ is

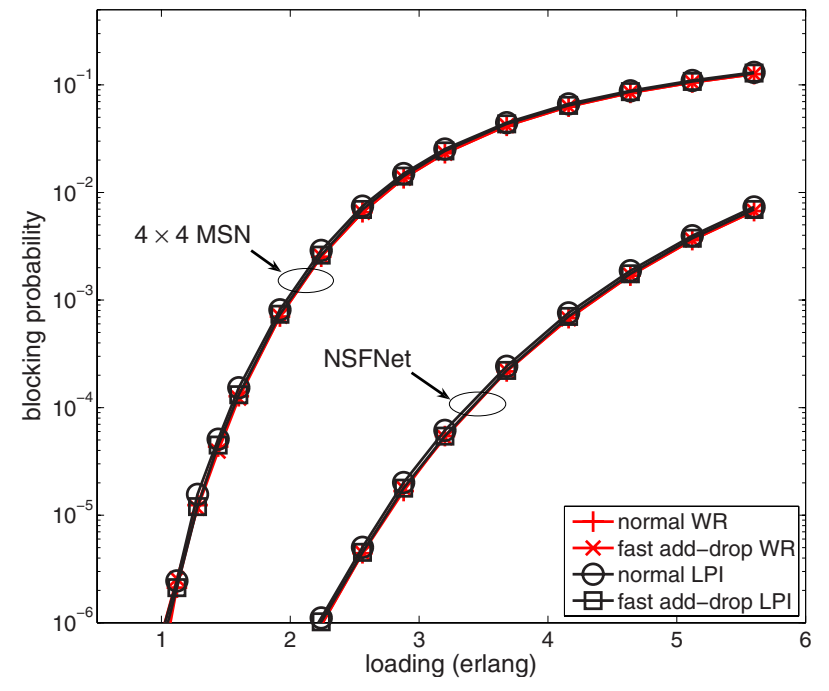

Fig. 6. (Color online) Blocking probabilities of 16 wavelength channel WR networks with LPIs on networks with topologies of the 4 $\times 4$ Manhattan Street network (MSN) [34] and NSFNet (Fig. 10).

not zero, communication can start only after the completion of all reconfigurations of the optical switches on the path even if all required wavelength channels on the links of the lightpath have been reserved. In normal WR networks (WR nodes with preinstalled TWCs for wavelength contention resolution purposes only), the call will be blocked if a direct lightpath connection cannot be set up. For WR networks with LPIs (basic WR nodes with external LPI devices as shown in Fig. 5), a call will be blocked only if the source and destination cannot be connected by either a direct lightpath or a sequence of lightpaths set up as described in Subsection II.A. In the simulation, the simple temporary lightpath arrangement in Subsection II.C is used. Figure 4 shows the detailed call blocking principle.

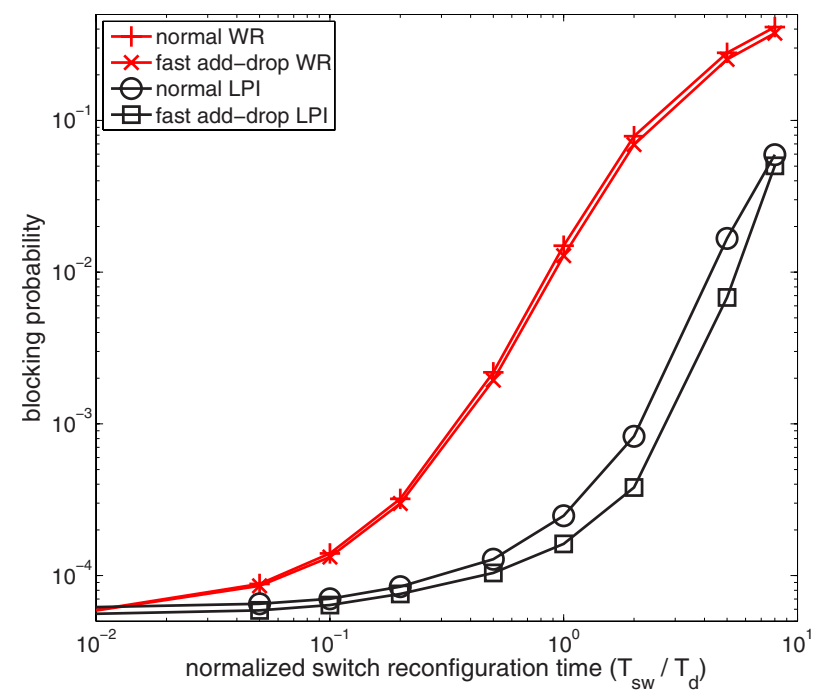

Fig. 7. (Color online) Switch reconfiguration time--blocking performance of WR networks with LPIs on networks with the topology of the NSFNet (Fig. 10). 


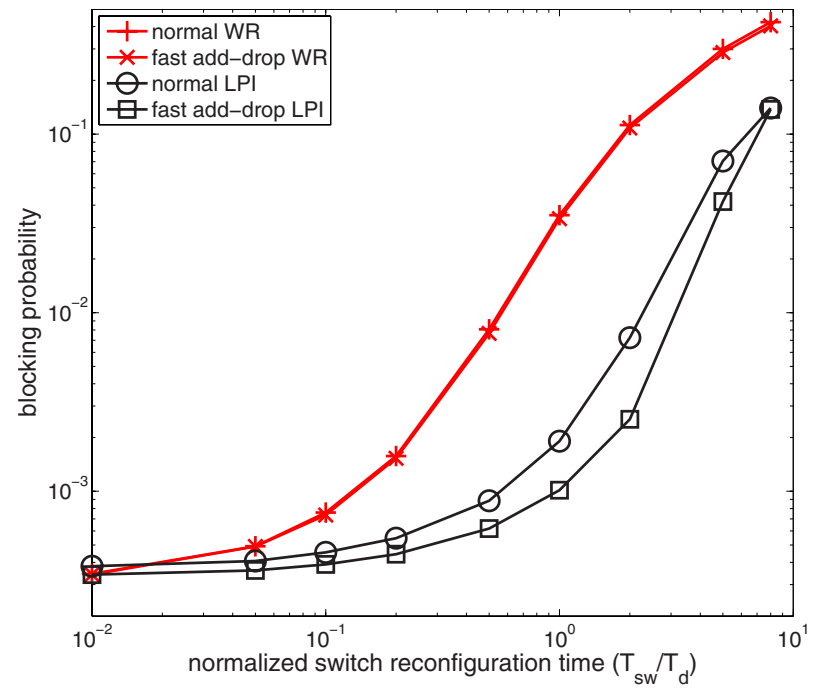

Fig. 8. (Color online) Switch reconfiguration time-blocking performance of WR networks with LPIs on a network with the topology of the $4 \times 4 \mathrm{MSN}$ [34].

In this paper, all simulations are run sufficiently long such that the $95 \%$ confidence intervals are less than $1 \%$ of the average values of the results. In Figs. $6-9$, the crosses and pluses represent the blocking probabilities of normal WR networks with and without the fast traffic add-drop function, respectively. The squares and circles represent the blocking probabilities of WR networks with LPIs and with and without the fast traffic add-drop function, respectively.

Figure 6 shows the comparison results when the networks are used in normal lightpath applications (traffic duration $T_{d}$ is much longer than that of the op-

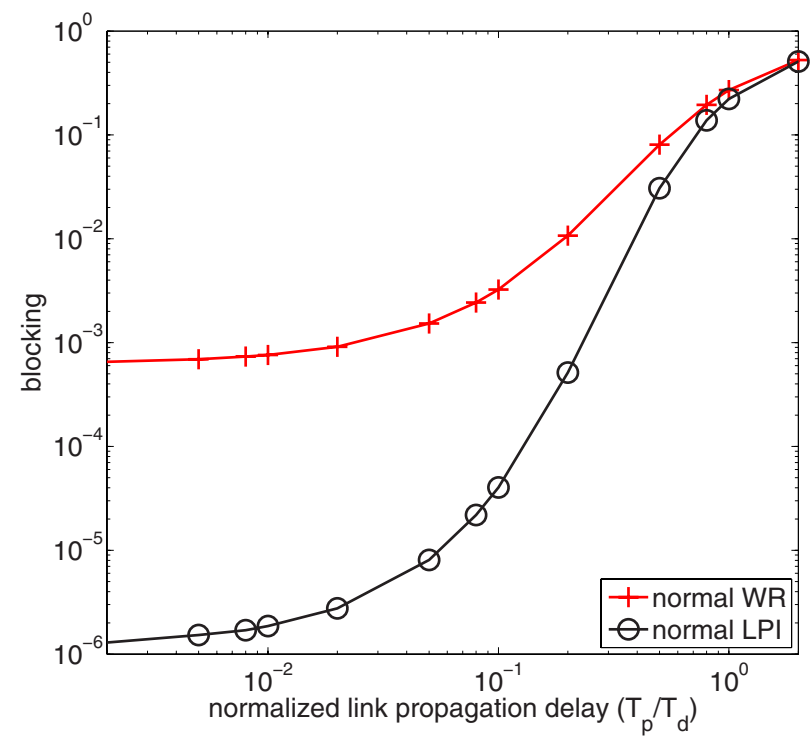

Fig. 9. (Color online) Link propagation delay-blocking performance of WR networks with LPIs on a network with the topology of Fig. $3(M=N=4)$. tical switch reconfiguration time $T_{s w}$ ). Hence, $T_{s w}$ is set to zero in Fig. 6 . The $x$ axis of Fig. 6 is the per-node offered loading in erlangs. The upper group of curves corresponds to the blocking probabilities for a $4 \times 4$ Manhattan Street network (MSN) [34], whereas the lower group of curves is for the NSFNet topology (Fig. 10). From Fig. 6 , we observe that WR networks with LPIs will not have performance improvement over WR networks without LPIs in normal lightpath applications if the fixed shortest path is used, regardless of the loading, network topology, and the fast traffic add-drop function. Obviously, using a sequence of lightpaths rather than a direct lightpath connection will not provide any benefit in a fixed path if the lightpath setup time (optical switch reconfiguration time) is not a critical factor. Since the overhead of traffic add-drop will be negligible if the traffic duration is long, the fast traffic add-drop function is also not useful in Fig. 6. We note that the performance of WR networks that also use their installed TWCs as LPIs is not different from that of WR networks that use the installed TWCs for wavelength contention resolution only. Figure 6 shows that WR networks using external LPI devices can have similar blocking performance even though basic WR nodes with external LPI devices in general have limitations in lightpath setup.

Figures 7 and 8 show the comparison when the traffic duration $T_{d}$ is not much longer than the optical switch reconfiguration time $T_{s w}$. Figure 7 shows the results of the NSFNet topology as shown in Fig. 10, and Fig. 8 shows that of the $4 \times 4$ MSN [34]. The $x$ axis in Figs. 7 and 8 is the ratio of $T_{s w} / T_{d}$. The per-node loadings in Figs. 7 and 8 are set to 3.2 and 1.76 erlangs such that the networks have blocking probabilities of $5.6 \times 10^{-5}$ and $3.3 \times 10^{-4}$, respectively, if the switch reconfiguration time $T_{s w}$ is zero. From Figs. 7 and 8 , we observe that WR networks with LPIs will have much better blocking performance than that of WR networks without LPIs if the traffic duration is short. For example, when $T_{s w}$ is equal to $T_{d}$, WR networks with LPIs but without the fast traffic add-drop function in Figs. 7 and 8 have blocking probabilities of

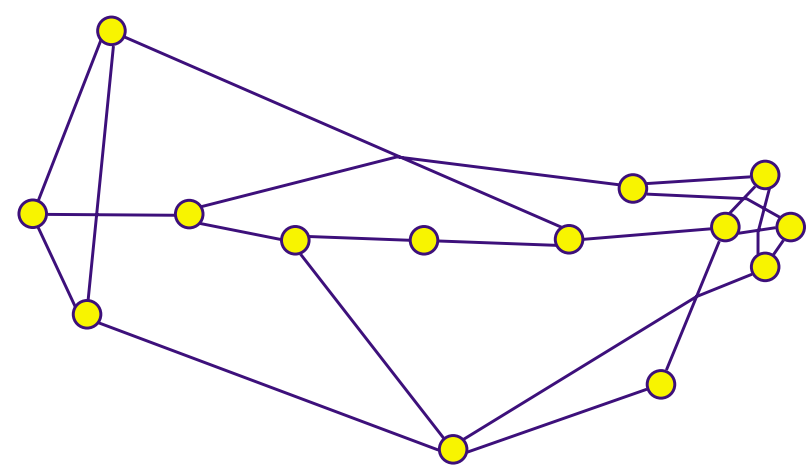

Fig. 10. (Color online) NSFNet (1991) network topology. The original map of the network is available from the Internet [35]. 
$2.5 \times 10^{-4}$ and $1.9 \times 10^{-3}$, respectively. The blocking probabilities are further reduced to $1.6 \times 10^{-4}$ and $1.1 \times 10^{-3}$, respectively, if the fast traffic add-drop function is used in WR networks with LPIs.

We observe that the freedom of setting up a sequence of lightpaths instead of only a single lightpath to connect the source to the destination can increase the reuse of existing internal connections in the optical switches of the nodes and reduces the requirement of switch reconfigurations. Hence, the blocking probability of WR networks with LPIs will increase much slower than that of WR networks without LPIs if $T_{s w} / T_{d}$ becomes large. With the fast traffic add-drop function, the traffic can be injected and removed from the system without passing through the optical switches of the source and destination. This further reduces the requirement of switch reconfiguration in WR networks with LPIs. In WR networks without LPIs, the direct lightpath connection requirement causes a large number of switch reconfigurations. We can only find insignificant performance improvement in Figs. 7 and 8 when the fast traffic add-drop function is used in WR networks without LPIs, i.e., the blocking probabilities in Figs. 7 and 8 are around 1.5 $\times 10^{-2}$ and $3.5 \times 10^{-2}$, respectively, with and without the fast traffic add-drop function.

Figures 7 and 8 show the ideal cases of LPI applications. In real networks, the link propagation delay between nodes may be large. Figure 9 shows the comparison results when $T_{s w}=T_{d}$ and the link propagation delay $T_{p}$ is not zero. The network topology shown in Fig. $3(M=N=4)$ is used for the simulations. The per-node loading in Fig. 9 is 0.32 erlangs. Since nodes $\mathrm{H}_{1}$ and $\mathrm{H}_{2}$ in Fig. 3 are pure routing nodes, the fast traffic add-drop function will not improve the network performance. We therefore only plot the blocking probabilities of the networks without the fast traffic adddrop function. The $x$-axis in Fig. 9 is the ratio of $T_{p} / T_{d}$. In Fig. 3, the round-trip time $T_{r t}$ from a source $\mathrm{S}_{i}$ to node $\mathrm{H}_{2}$ is $4 T_{p}$. As shown in Fig. $4, T_{r t}$ is another major component of the lightpath transmission overhead. The channel utilization improvement achieved by LPIs will become negligible if $T_{r t}$ dominates the overhead. This is confirmed by the results in Fig. 9. The blocking probabilities of networks with a normal lightpath and with LPI are $7.6 \times 10^{-4}$ and $1.9 \times 10^{-6}$, respectively, when $T_{p} / T_{d}$ is $10^{-2}$. Both kinds of networks, however, will have similar blocking probabilities if $T_{p} / T_{d}$ is large, e.g., $T_{p} / T_{d}=2$.

Figures 6 and 9 show the two kinds of networks for which LPI may be incapable of improving the system performance: (1) $T_{s w} / T_{d}$ is small, and (2) the link propagation delay is large $\left(T_{s w}\right.$ does not dominate the transmission overhead). For networks having large link propagation delay, one-way resource reservation schemes such as OBS have been proposed to eliminate the round-trip time overhead for better handling of short duration traffic [14-16]. Similarly, the throughput performance of such schemes will suffer if $T_{s w} / T_{d}$ is large. In principle, the LPI approach will improve the performance of these schemes. The detailed implementation requires further investigation. For networks in which normal lightpaths are applicable (small $T_{s w} / T_{d}$ ), LPI may still be useful for further improving the system performance if new data transportation services have been applied to improve the transmission bandwidth utilization, for example, with OTDMA.

\section{TIME-SLOTTED WR NETWORKS With LPIS}

In lightpath networks with OTDMA, a wavelength channel is divided into frames of time slots. Instead of taking up all the transmission capacity of a wavelength channel, new calls are assigned one or more time slots in each time frame. The source and destination nodes will transmit/receive data only at the assigned time slots. Since multiple source and destination pairs can share a single wavelength channel, OTDMA networks can utilize the fiber transmission bandwidth more efficiently than non-OTDMA networks. Unlike OPS and OBS networks [12-16], OTDMA networks are still circuit switched, i.e., call setup must be completed before data transmission can start. It reduces the negative impacts caused by outdated link status information that can greatly degrade the system performance in one-way resource reservation schemes such as OBS [14-16]. We will have sufficient time to process and update the information during call setup if the call transmission duration is long. Large link propagation delay between nodes will no longer be a concern. Without upgrading the WR nodes to have slot-by-slot switchable fast optical switches, however, all data slots in the same wavelength can only be accessed by the nodes on the same lightpath. Using LPIs, data in different time slots of the same wavelength channel can be routed to destinations on different lightpaths. Hence, the transmission bandwidth utilization is further improved.

Finding the proper way to avoid data collisions when data are switched between lightpaths by LPIs will be the prerequisite requirement if we want to provide new data transportation services in WR networks with LPIs. As the wavelength channels in OTDMA networks are time slotted, the cooperation between data transmissions in lightpaths can be achieved by reserving the appropriate time slots in the links on the path. In the following, we will demonstrate the application of LPIs in WR networks to provide the OTDMA time-slotted service and its performance when compared with time-slotted WR networks without LPIs. 


\section{A. Channel Assignment in Time-Slotted WR Networks} With LPIs

As the data forwarding may need to pass through a sequence of lightpaths as we discussed in Subsection II.A, the choosing of the appropriate time slots and the sequence of lightpaths will be important for system performance. We assume that the path for data forwarding is fixed, e.g., the minimum hop routing. To determine the proper time slots and lightpaths for data routing, we need to first list all possible sequences of lightpaths that can be used to connect the source to the destination using LPIs. We define $\mathbf{S}(s, d)$ as the set of all valid lightpath sequences between source $s$ and destination $d$. For one $(s, d)$ sourcedestination node pair that has a path length of $L$ links, we further define $\mathbf{S}_{h}(s, d)$ as the subset of $\mathbf{S}(s, d)$ in which all the sequences have $h$ lightpaths, where $2 \leqslant h \leqslant L-1$. The steps to construct the set $\mathbf{S}(s, d)$ are as follows:

1. Choose $k$ links $j_{1}, j_{2}, \ldots$, and $j_{k}$ between the 2 nd and $(L-1)$ th links of the path.

2. Find $\mathbf{L}_{g}$ : the set of lightpaths passing all links in link segment $\left(j_{g}, j_{g-1}\right)$ for $g=0,1, \ldots, k$, where $j_{0}$ and $j_{k+1}$ are assumed to be the first and last links in the path.

3. The set $\mathbf{S}_{h}(s, d)$ can be formed by exhausting the combinations of $h$ lightpaths, with one lightpath from each of the sets $\mathbf{L}_{0}, \mathbf{L}_{1}, \ldots, \mathbf{L}_{h-1}$.

4. Repeat steps 1 to 3 for $k=1,2, \ldots, L-2$ to compute all $\mathbf{S}_{h}(s, d)$ sets.

5. Compute the $\mathbf{S}(s, d)$ by $\mathbf{S}(s, d)=\cup_{h=2}^{L-1} \mathbf{S}_{h}(s, d)$.

As an example, Fig. 11 shows a five-node fourwavelength-per-fiber WR network with LPIs. The small rectangles in front of the nodes are external LPI devices. We assume the fast traffic add-drop function. Five lightpaths $P_{1}$ to $P_{5}$ have already been set up in the network. Using the procedure described above, $\mathbf{S}_{2}(1,5)$ of Fig. 11 is $\left\{\mathrm{P}_{1} \mathrm{P}_{4}, \mathrm{P}_{1} \mathrm{P}_{5}{ }^{(3)}, \mathrm{P}_{2} \mathrm{P}_{1}{ }^{(3)}, \mathrm{P}_{2} \mathrm{P}_{4}\right.$, $\left.\mathrm{P}_{2} \mathrm{P}_{5}{ }^{(3)}, \mathrm{P}_{3} \mathrm{P}_{1}{ }^{(2)}, \mathrm{P}_{3} \mathrm{P}_{5}{ }^{(2)}\right\}$. The superscript shows the possible choices of data forwarding along the lightpath sequence. For example, $\mathrm{P}_{1} \mathrm{P}_{5}^{(3)}$ represents data that can be forwarded from node 1 to node 5 along lightpath $\mathrm{P}_{1}$ and lightpath $\mathrm{P}_{5}$ with choices of switching data from $\mathrm{P}_{1}$ to $\mathrm{P}_{5}$ using the LPIs at nodes 2,3 , and 4 . Hence, the actual sizes of $\mathbf{S}_{2}(1,5), \mathbf{S}_{3}(1,5)$, and $\mathbf{S}_{4}(1,5)$ are 15,45 , and 47 , respectively. Since the

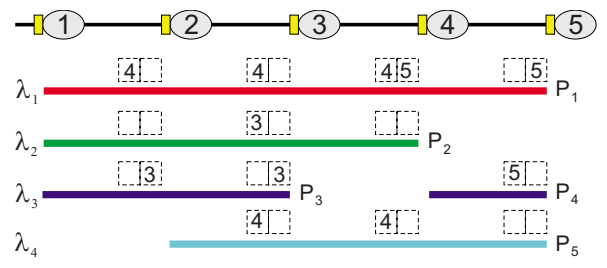

Fig. 11. (Color online) Five-node time-slotted WR network with LPIs. large number of combinations of time slots and lightpaths will render the optimal solution computationally infeasible for large networks, a heuristic is used to assign the time slots after $\mathbf{S}(s, d)$ is obtained.

Channel Assignment Heuristic: We assume that there are $W$ wavelength channels per fiber link and $T$ time slot channels per wavelength channel. Since we have wavelength converters (inside the LPIs) but no time slot interchanger in the network, all time slot channels assigned to a call on different links must be located in the same position in the $T$ time slot frame, but they can belong to different wavelength channels. As shown in Fig. 11, the dashed squares above the lightpaths represent the first and second (from left to right) time slot channels. The numbers in the squares are the destinations of the reserved time slot channels. Hence, there are six successfully connected calls $(1 \rightarrow 3),(1 \rightarrow 4),(2 \rightarrow 3),(2 \rightarrow 4),(3 \rightarrow 5)$, and $(4 \rightarrow 5)$. Assume that a new call $(1 \rightarrow 5)$ arrives. No time slot channel in any single lightpath will be available to send data from node 1 to node 5. Although we can modify the lightpaths such as lengthening lightpath $\mathrm{P}_{2}$ and lightpath $\mathrm{P}_{5}$ to cover all nodes, it is not preferred. Using the LPI approach, we can send the data with one of the four link-by-link lightpath switching sequences $\left\{\mathrm{P}_{2}, \mathrm{P}_{3}, \mathrm{P}_{2}, \mathrm{P}_{1}\right\},\left\{\mathrm{P}_{2}, \mathrm{P}_{3}, \mathrm{P}_{2}, \mathrm{P}_{5}\right\},\left\{\mathrm{P}_{3}, \mathrm{P}_{3}, \mathrm{P}_{2}\right.$, $\left.\mathrm{P}_{1}\right\}$, and $\left\{\mathrm{P}_{3}, \mathrm{P}_{3}, \mathrm{P}_{2}, \mathrm{P}_{5}\right\}$ if we use the first time slot channel. There will be $24(2 \times 3 \times 2 \times 2)$ sequences if the second time slot channel is used. Instead of checking all lightpath sequences, we assign the time slot and wavelength channels link by link by maximizing the residual capacity on each link and minimizing the optical switch reconfigurations in the intermediate nodes.

The time-slotted WR networks with LPIs first search for a suitable time slot channel index $t$ and then assign a new call link by link with the smallest index lightpath that has the free $t$ th time slot channel. We assume that the source node $s$ has the information of all links $j_{1}, j_{2}, \ldots$, and $j_{L}$ on the path to the destination $d$. Source node $s$ first constructs the set $\mathbf{Q}(s, d)$, which consists of the direct lightpaths and all lightpaths in the lightpath sequences connecting nodes $s$ and $d$. Hence, $\mathbf{Q}(1,5)$ of Fig. 11 is $\left\{\mathrm{P}_{1}, \mathrm{P}_{2}, \mathrm{P}_{3}, \mathrm{P}_{4}, \mathrm{P}_{5}\right\}$. Node $s$ will also construct a set $\mathbf{Q}_{p}(s, d)$, which includes all possible lightpaths after we have made the lightpath modifications to increase the available time slot channels in each link for the connection. For example, we may extend the existing lightpaths $\mathrm{P}_{2}$ and $\mathrm{P}_{5}$ in Fig. 11 to cover all links as $\mathrm{P}_{20}$ and $\mathrm{P}_{50}$. Similarly, we may add a new lightpath segment to combine the two existing lightpaths $\mathrm{P}_{3}$ and $\mathrm{P}_{4}$ to form a new lightpath $\mathrm{P}_{34}$ to cover all links. Hence, $\mathbf{Q}_{p}(1,5)$ of Fig. 11 is $\left\{\mathrm{P}_{1}, \mathrm{P}_{20}, \mathrm{P}_{34}, \mathrm{P}_{50}\right\}$. After obtaining $\mathbf{Q}_{p}(s, d)$, node $s$ will try to find a time slot channel $t$ with the largest capacity weighing $f_{t}$. Let $x_{k, i}$ be the number of free 
time slot channels with index $k$ in link $j_{i}$ that belong to the lightpaths of the set $\mathbf{Q}_{p}(s, d)$. We define $f_{t}$ as $f_{t}$ $=\min _{i=1}^{L}\left(x_{t, i}\right)$. If $\max _{t=1}^{T}\left(f_{t}\right)=0$, node $s$ will block the new call immediately. Otherwise, the $t$ th time slot channel of a lightpath of each link on the path will be assigned to the new call as we have described earlier. The available lightpaths of each link will be first selected from $\mathbf{Q}(s, d)$ and then $\mathbf{Q}_{p}(s, d)$ to reduce the modifications of lightpaths (reconfigurations of the optical switches). In Fig. 11, $x_{1, i}=\{3,1,2,3\}$ and $x_{2, i}=\{3,3,3,3\}$ for $i$ $=1,2,3,4$, respectively. Because $f_{1}=1$ and $f_{2}=3$, the second time slot channel is used. The sequence of lightpaths that is used on each link is $\left\{\mathrm{P}_{1}, \mathrm{P}_{1}, \mathrm{P}_{2}, \mathrm{P}_{4}\right\}$.

\section{B. Blocking Performance of the Time-Slotted WR Networks With LPIs}

To demonstrate the advantage of LPI, we use simulations to compare the blocking performance between the time-slotted WR networks with LPIs and those without LPIs. The simulation settings are similar to those in Subsection II.D with a time slot channel being required by each new call. A call will be blocked immediately if any link on the path cannot provide a time slot using the predefined time slot searching algorithm. For the time-slotted WR networks with LPIs, the heuristic presented in Subsection III.A is used to search and reserve the time slots in different links. For the time-slotted WR networks without LPIs, we use the exhaustive search approach to find the first available time slot and wavelength channel on all links of the path. In both networks, the reserved time slot channels will be released immediately once the call is terminated.

Figures 12-15 compare the blocking performance of

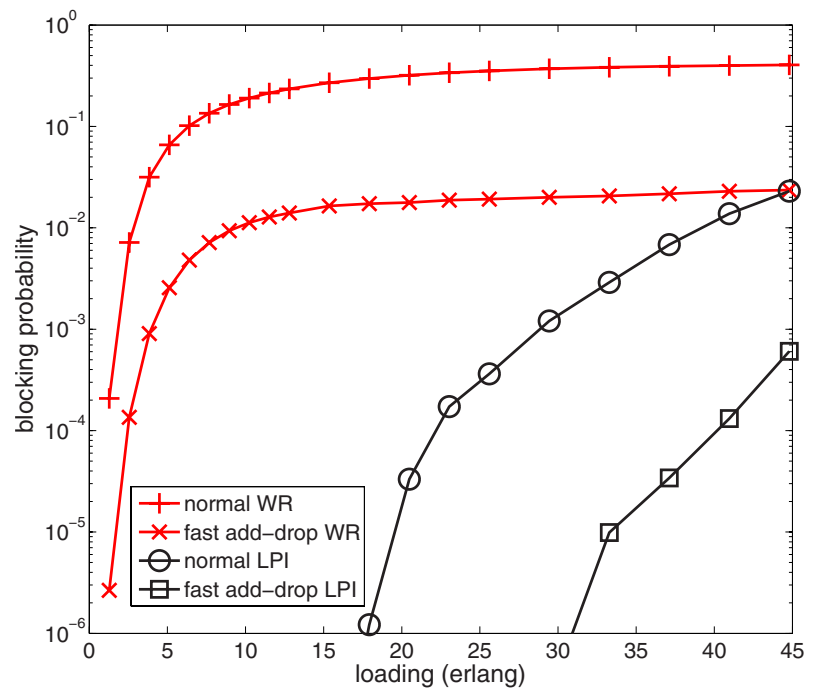

Fig. 12. (Color online) Blocking probabilities of the time-slotted WR networks with LPIs on a network with the topology of the NSFNet (Fig. 10). There are 8 wavelength channels per fiber and 16 time channels per wavelength.

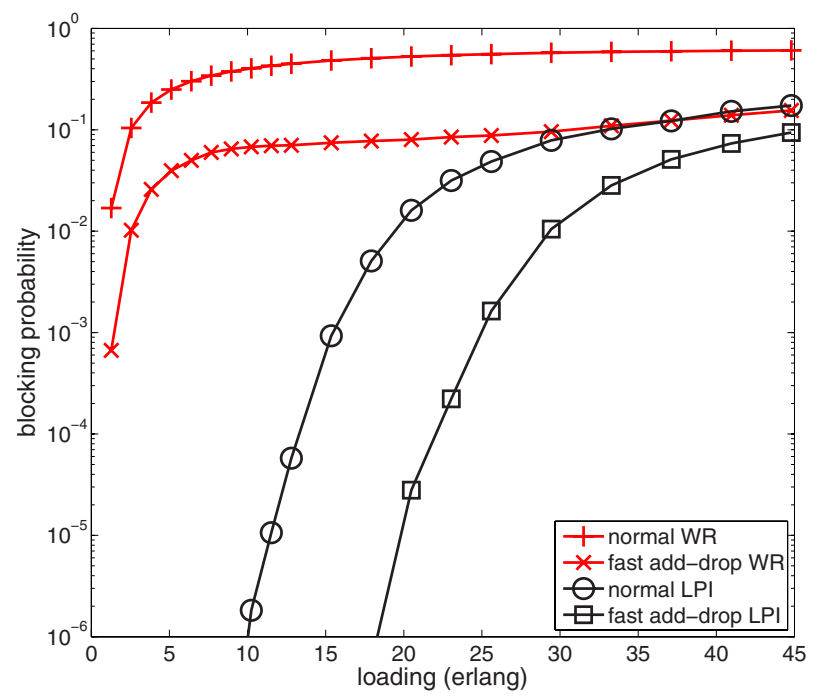

Fig. 13. (Color online) Blocking probabilities of the time-slotted WR networks with LPIs on a network with the topology of the 4 $\times 4$ MSN [32]. There are 8 wavelength channels per fiber and 16 time channels per wavelength.

the time-slotted WR networks with LPIs (basic WR nodes with external LPI devices as shown in Fig. 5) and that of the time-slotted normal WR networks (WR nodes with preinstalled TWCs for wavelength contention resolution purposes only). The network topology used for Figs. 12 and 14 is the NSFNet shown in Fig. 10 and that for Figs. 13 and 15 is the $4 \times 4$ MSN [32]. The wavelength channels per fiber $(W)$ and time channels per wavelength $(T)$ used for Figs. 12 and 13 are $W=8$ and $T=16$, and those for Figs. 14 and 15 are $W$ $=16$ and $T=8$, respectively. In the figures, the $x$ axis is the average offered traffic load at a node. The lines with crosses and pluses are the blocking probabilities

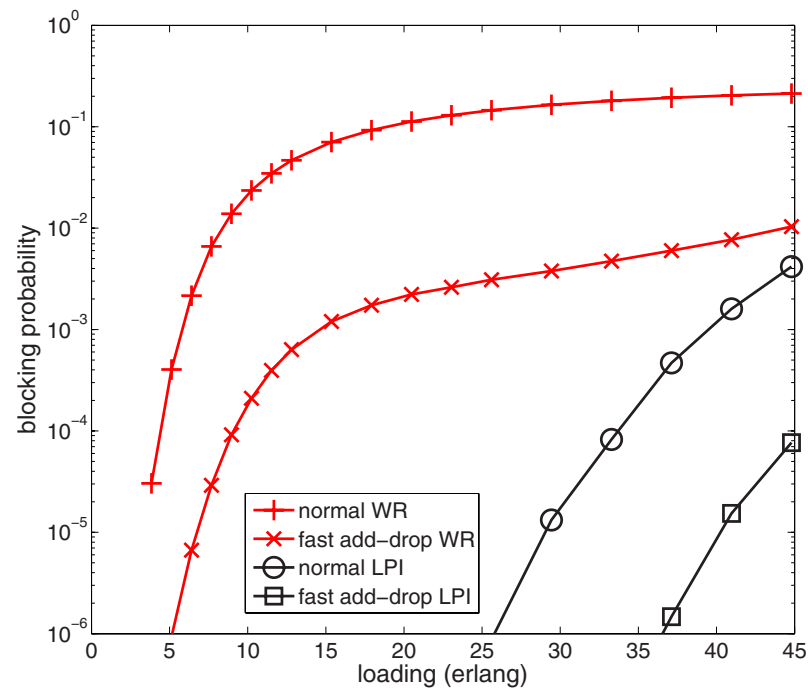

Fig. 14. (Color online) Blocking probabilities of the time-slotted WR networks with LPIs on a network with the topology of the NSFNet (Fig. 10). There are 16 wavelength channels per fiber and 8 time channels per wavelength. 


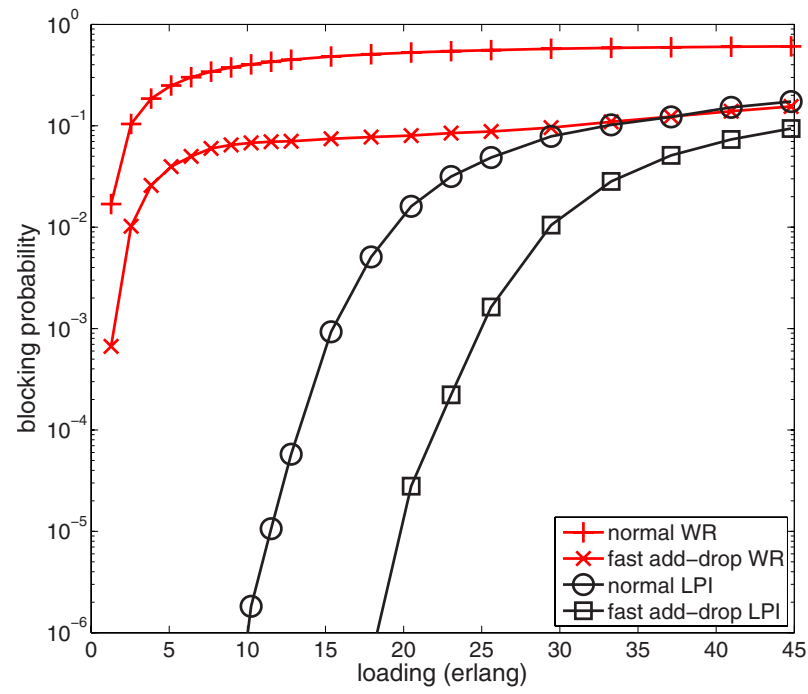

Fig. 15. (Color online) Blocking probabilities of the time-slotted WR networks with LPIs on a network with the topology of the 4 $\times 4 \mathrm{MSN}$ [32]. The are 16 wavelength channels per fiber and 8 time channels per wavelength.

of the time-slotted normal WR networks with and without the fast traffic add-drop function, respectively. The lines with squares and circles are those of the time-slotted WR networks with LPIs and with and without the fast traffic add-drop function, respectively.

From the figures, we observe that time-slotted WR networks with LPIs have much better blocking performance whether the fast traffic add-drop function is used or not. In most cases, however, time-slotted WR networks with LPIs but without the fast traffic adddrop function can even have lower blocking probability than time-slotted normal WR networks with the fast traffic add-drop function. Even though the combination of a basic node and an external LPI device puts more limitations on lightpath setup, the features of LPI have significantly improved the time slot channel utilization of WR networks. For example, the fast traffic add-drop function enables all nodes, not only the terminal nodes, on the lightpath to read/write data to time slots on the lightpath. The function reduces the blocking probability in both kinds of networks, but the reduction is much more significant in time-slotted WR networks with LPIs. From simulation results (not all being shown here), we observe that the blocking probability decreases with an increasing number of available wavelength channels and time slot channels. However, the decrease is more sensitive to the increase in wavelengths than in time slots. For example, the blocking probabilities in Figs. 14 and 15 are smaller than those of Figs. 12 and 13.

\section{CONCLUSION}

We observe that WR networks will likely be the dominant optical networks in the near future. To pro- tect the investment in infrastructure, it is important to develop new optical data transportation schemes that can utilize WR networks to provide fast data switching services.

In this paper, we have observed that the TWCs that are traditionally installed in the WR nodes for wavelength contention resolution purposes during lightpath setup can be further utilized as the fast data switching devices to provide fast data switching between lightpaths. As the TWCs become the interchanges of data units between lightpaths, we can route data through a sequence of lightpaths from source to destination if a direct single lightpath connection is not available or if we want to minimize the overhead of setting up new lightpaths. Since TWCs can have a tuning time of picoseconds, one can greatly improve the performance of WR networks. Ideally, the WR node's preinstalled TWCs can be used as LPI without significant optical hardware upgrade. From simulation results, we show that WR networks with LPIs can have much lower blocking probability than WR networks without LPIs if the traffic duration is short.

We have also demonstrated how LPI can be used to provide new data transportation services such as the OTDMA time-slotted service in WR networks. Using LPIs, data in different time slots on the same wavelength channel can be routed to destinations on different lightpaths. Simulation results show that the LPI approach can significantly improve the system blocking performance. Different implementations of WR networks with LPIs such as external LPI devices and the fast traffic add-drop function are also discussed.

\section{ACKNOWLEDGMENT}

This research was supported by a grant from the Hong Kong Polytechnic University (project number 1-0512) and the University of Hong Kong Strategic Research Theme of Information Technology.

\section{REFERENCES}

[1] A. Gladisch, R.-P. Braun, D. Breuer, A. Ehrhardt, H.-M. Frisel, M. Jaeger, R. Leppla, M. Schneiders, S. Verbeck, W. Weiershausen, and F.-J. Westphal, "System and core network architecture," Proc. IEEE, vol. 94, no. 5, pp. 869-981, 2006.

[2] J. Berthold, A. A. M. Saleh, L. Blair, and J. M. Simmons, "Optical networking: past, present, and future," J. Lightwave Technol., vol. 26, no. 9, pp. 1104-1118, 2008.

[3] I. Chlamtac, A. Ganz, and G. Karmi, "Lightpath communications: an approach to high bandwidth optical WANs," IEEE Trans. Commun., vol. 40, no. 7, pp. 1171-1182, 1992.

[4] K. C. Lee and V. O. K. Li, "A wavelength-convertible optical network," J. Lightwave Technol., vol. 11, no. 5, pp. 962-970, 1993.

[5] N. Ghani, S. Dixit, and T. S. Wang, "On IP-over-WDM integration,” IEEE Commun. Mag., vol. 38, no. 3, pp. 72-84, 2000. 
[6] N. U. Kim and M. Kang, "Traffic share-based multicast scheduling for broadcast video delivery in shared-WDM-PONs," $J$. Lightwave Technol., vol. 25, no. 9, pp. 2814-2827, 2007.

[7] S. Sankaranarayanan and S. Subramaniam, "Comprehensive performance modeling and analysis of multicasting in optical networks," IEEE J. Sel. Areas Commun., vol. 21, no. 9, pp. 1399-1413, 2003.

[8] W. E. Leland, M. S. Taqqu, W. Willinger, and D. V. Wilson, "On the self-similar nature of Ethernet traffic (extended version)," IEEE / ACM Trans. Netw., vol. 2, no. 1, pp. 1-15, 1994.

[9] T. Karagiannis, M. Molle, and M. Faloutsos, "Long-range dependence ten years of Internet traffic modeling," Inf. Manage., vol. 8, no. 5, pp. 57-64, 2004.

[10] C. S. Xin, C. M. Qiao, and S. Dixit, "Traffic grooming in mesh WDM optical networks-performance analysis," IEEE J. Sel. Areas Commun., vol. 22, no. 9, pp. 1658-1669, 2004.

[11] S. Huang, R. Dutta, and G. N. Rouskas, "Traffic grooming in path, star, and tree networks: complexity, bounds, and algorithms," IEEE J. Sel. Areas Commun., vol. 24, no. 4, pp. 66-82, 2006.

[12] T. S. El-Bawab and J.-D. Shin, "Optical packet switching in core networks: between vision and reality," IEEE Commun. Mag., vol. 40, no. 9, pp. 60-65, 2002.

[13] D. J. Blumenthal, J. E. Bowers, L. Rau, H.-F. Chou, S. Rangarajan, W. Wang, and K. N. Poulsen, "Optical signal processing for optical packet switching networks," IEEE Commun. Mag., vol. 41, no. 2, pp. S23-S29, 2003.

[14] C. Qiao and M. Yoo, "Optical burst switching (OBS)—a new paradigm for an optical Internet," J. High Speed Networks, vol. 8, pp. 69-84, 1999.

[15] J. S. Tuner, "Terabit burst switching," J. High Speed Networks, vol. 8, pp. 3-16, 1999.

[16] J. Y. Wei and R. I. McFarland, Jr., "Just-in-time signaling for WDM optical burst switching networks," J. Lightwave Technol., vol. 18, no. 12, pp. 2019-2037, 2000.

[17] M. Düser and P. Bayvel, "Analysis of a dynamically wavelength-routed optical burst switched network architecture," J. Lightwave Technol., vol. 20, no. 4, pp. 574-585, 2002.

[18] M. L. Rocha, S. M. Rossi, M. R. X. Barros, L. Pezzollo, J. B. Roselem, M. F. Oliveira, A. Paradisi, T. Kauppinen, and A. Gavler, "Amplifier placement in metro-scaled wavelengthrouted network," Electron. Lett., vol. 39, no. 3, pp. 302-304, 2003.

[19] K. Noguchi, Y. Koike, H. Tanobe, K. Harada, and M. Matsuoka, "Field trial of full-mesh WDM network (AWG-STAR) in metropolitan/local area," J. Lightwave Technol., vol. 22, no. 2, pp. 329-336, 2004.

[20] T. Lehman, J. Sobieski, and B. Jabbari, "DRAGON: a framework for service provisioning in heterogeneous grid networks," IEEE Commun. Mag., vol. 44, no. 3, pp. 84-90, 2006.

[21] J. Kim, C. J. Nuzman, B. Kumar, D. F. Lieuwen, J. S. Kraus, A. Weis, C. P. Lichtenwalner, A. R. Parpazian, R. E. Frahm, N. P. Basavanhally, D. A. Ramsey, V. A. Aksyule, F. Pardo, M. S. Simon, V. Lifton, H. R. Chan, M. Hauies, A. Gasparyan, H. R. Shea, S. Arney, C. A. Bolle, P. R. Kolodne, R. Ryf, D. T. Neilson, and J. V. Coate, " $1100 \times 1100$ port MEMS-based optical crossconnect with 4-dB maximum loss," IEEE Photon. Technol. Lett., vol. 15, no. 11, pp. 1537-1539, 2003.

[22] S. J. B. Yoo, "Optical packet and burst switching technologies for the future photonic Internet," J. Lightwave Technol., vol. 24, no. 12, pp. 4468-4492, 2006.

[23] S. Sygletos, I. Tomaos, and J. Leuthold, "Technological challenges on the road toward transparent networking," J. Opt. Netw., vol. 7, no. 4, pp. 321-350, 2008.

[24] P. Bernasconi, L. Zhang, W. Yang, N. Sauer, L. L. Buhl, J. H. Sinsky, I. Kang, S. Chandrasekhar, and D. T. Neilson, "Monolithically integrated $40-\mathrm{Gb} / \mathrm{s}$ switchable wavelength converter," J. Lightwave Technol., vol. 24, no. 1, pp. 71-76, 2006.

[25] Y. Liu, E. Tangdiongga, Z. Li, H. de Waardt, A. M. J. Koonen, G. D. Khoe, X. Shu, I. Bennion, and H. J. S. Dorren, "Error-free $320-\mathrm{Gb} / \mathrm{s}$ all-optical wavelength conversion using a single semiconductor optical amplifier," J. Lightwave Technol., vol. 25, no. 1, pp. 103-108, 2007.

[26] K. Vlachos, C. Raffaelli, S. Aleksic, N. Andriolli, D. Apastoloponlas, H. Auramoponlos, D. Erasme, D. Klonidis, M. N. Petersen, M. Scaffardi, K. Schutze, M. Spiropoulov, S. Sygletos, I. Tunkos, C. Vazquez, O. Zouraraki, and F. Neri, "Photonics in switching: enabling technologies and subsystem design," $J$. Opt. Netw., vol. 8, no. 5, pp. 404-428, 2009.

[27] T. K. Chan, N. J. Karp, R. Jiang, N. Alic, S. Radic, C. F. Marki, and J. G. Ford, "1092 channel 2-D array demultiplexer for ultralarge data bandwidth," J. Lightwave Technol., vol. 25, no. 3, pp. 719-725, 2007.

[28] A. Tajima, N. Kitamura, S. Takahashi, S. Kitamura, Y. Maeno, Y. Suemurg, and N. Nenmi, "10-Gb/s/port gated divider passive combiner optical switch with single-mode-to-multimode combiner," IEEE Photon. Technol. Lett., vol. 10, no. 1, pp. 162164, 1998.

[29] R. Chen, H. Zhou, G. Jiang, Y. Sun, Y. Hao, J. Yang, M. Wang, and X. Jiang, "A proposal of zero leakage-loss passive optical combiner based on nonreciprocal waveguide," IEEE Photon. Technol. Lett., vol. 21, no. 20, pp. 1493-1495, 2009.

[30] Y. Jaouen, L. du Mouza, D. Barbier, J. M. Delavaux, and P. Bruno, "Eight-wavelength Er-Yb doped amplifier: combiner/ splitter planar integrated module," IEEE Photon. Technol. Lett., vol. 11, no. 9, pp. 1105-1107, 1999.

[31] C. Y. Li and P. K. A. Wai, "External wavelength contention resolution for optical crossconnects," in Proc. of Opto-Electronics and Communications Conf. (OECC 2009), 2009, paper ThB6.

[32] M. Kodialam, T. V. Lakshman, J. B. Orlin, and S. Sengupta, "Oblivious routing of highly variable traffic in service overlays and IP backbones," IEEE / ACM Trans. Netw., vol. 17, no. 2, pp. 459-472, 2009.

[33] R. Razavi and K. Guild, "Multiconstraints fuzzy-logic-based scheduling algorithm for passive optical networks," J. Opt. Netw., vol. 8, no. 4, pp. 346-357, 2009.

[34] N. F. Maxemchuk, "Routing in Manhattan Street network," IEEE Trans. Commun., vol. 35, no. 5, pp. 503-512, 1987.

[35] ftp://ftp.uu.net/inet/maps/nsfnet/. 